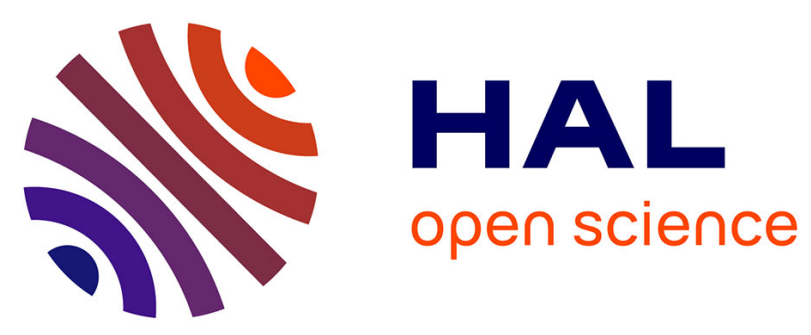

\title{
The SMARTLAM 3D-I Concept: Design of Microsystems from Functional Elements Fabricated by Generative Manufacturing Technologies
}

Markus Dickerhof, Daniel Kimmig, Raphael Adamietz, Tobias Iseringhausen, Joel Segal, Nikola Vladov, Wilhelm Pfleging, Maika Torge

\section{To cite this version:}

Markus Dickerhof, Daniel Kimmig, Raphael Adamietz, Tobias Iseringhausen, Joel Segal, et al.. The SMARTLAM 3D-I Concept: Design of Microsystems from Functional Elements Fabricated by Generative Manufacturing Technologies. 7th International Precision Assembly Seminar (IPAS), Feb 2014, Chamonix, France. pp.147-160, 10.1007/978-3-662-45586-9_19 . hal-01260754

\section{HAL Id: hal-01260754 \\ https://inria.hal.science/hal-01260754}

Submitted on 22 Jan 2016

HAL is a multi-disciplinary open access archive for the deposit and dissemination of scientific research documents, whether they are published or not. The documents may come from teaching and research institutions in France or abroad, or from public or private research centers.
L'archive ouverte pluridisciplinaire HAL, est destinée au dépôt et à la diffusion de documents scientifiques de niveau recherche, publiés ou non, émanant des établissements d'enseignement et de recherche français ou étrangers, des laboratoires publics ou privés. 


\title{
The SMARTLAM 3D-I Concept: Design of Microsystems from functional elements fabricated by generative manufacturing technologies
}

\author{
Markus Dickerhof ${ }^{1}$, Daniel Kimmig ${ }^{1}$, Raphael Adamietz ${ }^{2}$, Tobias Iseringhausen ${ }^{2}$, \\ Joel Segal ${ }^{3}$, Nikola Vladov ${ }^{3}$, Wilhelm Pfleging ${ }^{4}$, Maika Torge ${ }^{5}$ \\ ${ }^{1}$ Institute for Applied Computer Science, Karlsruhe Institute of Technology, Karlsruhe, DE, \\ ${ }^{2}$ Fraunhofer Institute for Process Automation, Stuttgart, DE, \\ ${ }^{3}$ Manufacturing Research Division, Faculty of Engineering, University of Nottingham, Nottingham, UK \\ ${ }^{4}$ Institute for Applied Materials Karlsruhe Institute of Technology, Karlsruhe, DE, \\ ${ }^{5}$ Karlsruhe Nano Micro Facility, H.-von-Helmholtz-Platz 1, 76344 Egg.-Leopoldshafen, DE
}

\begin{abstract}
Generative manufacturing technologies are gaining more and more of importance as key enabling technologies in future manufacturing, especially when a flexible scalable manufacturing of small medium series of customized parts is required. The paper describes a new approach for design and manufacturing of complex three dimensional components building on a combination of additive manufacturing and e-printing technologies, where the micro component is made up of stacks of functionalized layers of polymer films. Special attention will be paid to the "3-d" modeling approach, requested to support the applicaton developer through provision of design rules for this integrated manufacturing concept. Both, the application concept as well as the related equipment and manufacturing integration currently are currently developed further in the project SMARTLAM, funded by the European Commission.
\end{abstract}

Keywords: flexible scalable manufacturing, smart manufacturing, additive manufacturing, printing technologies.

\section{Introduction}

Today's fabrication methods for micro and nanotechnology enabled devices require expensive tooling and long turnaround times, making empirical, performance-based modifications to the product design expensive and time consuming. Thus till to date are often limited in their flexibility, so that complex devices, that incorporate on-board valves, membranes, discrete parts, or electrodes, cannot be developed or adapted without considerable expense in molds and assembly fixtures.

These boundary conditions create a barrier to the development of small to mediul series of complex and higher functionality devices, where the costbenefit ratio of incorporating functionality is too risky for the typical laboratory, diagnostic or medical device developer. To bridge the gap between a high volume production with specialized equipment and a - until today - not efficient production of medium series, SME's need to find other, more flexible and scalable approaches to produce microsystems in high volumes.

The solution proposed by the EC-funded project SMARTLAM and presented in this paper builds on a modular, flexible, scalable scenario combining state of the art developments in technologies and materials:

- Rapid prototyping technologies in a wider sense and laminated object manufacturing (LOM) in the narrow sense - an established rapid prototyping technology building on layer by layer lamination of functionalised film sheets with different material properties is in the focus of the activities [1].

- Printing technologies, where aerosol-jet printing is in the specific focus of the project allowing for an efficient and precise manufacturing of conductive tracks, electrodes, etc.

Novel polymer film materials with advanced material properties such as anisotropic conductive film or effects arising from combinations of composite sheets will be combined with state of the art, scalable $3 \mathrm{D}$ printing, structuring and welding technologies as well as the usage of.

Both technologies will be integrated in a modular manufacturing environment allowing for the production of complete 3D Microsystems.

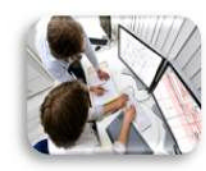

3D-I Modelling

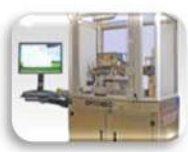

3D- Aerosol

inkjet printing

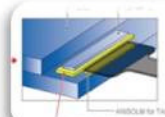

Materials with advanced properties

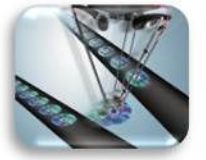

3D-I compatible 3D-I compatible

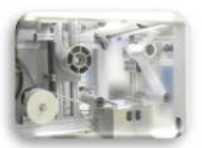

Handling

of polymer films

Smartlam Modules

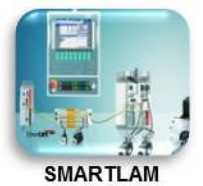

daptive control an

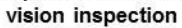

Fig. 1. SMARTLAM enabling technologies. 
The approach proposed by SMARTLAM is designed to address the manufacturing of small medium series of micro enabled components, in contrary to the research field of roll-to-roll manufacturing focussing on a high throughput manufacturing of e-printed devices such as flexible electronics, flat panel displays or organic photovoltaics [2].

The SMARTLAM design approach builds on the assumption that most applications can be designed using modular building blocks with dedicated process sequences for each functional element - the 3 dimensional integration (3D-I) approach.

To allow for a better use of the new capabilities arising from this 3D-Integration a testbed will be set up and evaluated by two SME companies in the field of bioanalytics and lighting application. The companies are acting as potential customers, whose application requirements will be providing input to the 3D-I approach from a technical and economical perspective.

\section{Application oriented modeling elements of the SMARTLAM 3D-I approach}

To facilitate the development of new applications the SMARTLAM consortium introduced a modelling hierarchy allowing for structuring of the different levels of detailing and the mapping of technological capabilities, after the initial function requirements have been clarified and a first decision for a specific design has been made.

Over the first months the focus of the discussions was on the identification of an initial set of functional elements which will become expanded during the project. Sets of related process sequences for manufacturing are currently identified for each of these elements These specific process chains support the implementation of a specific functional element, representing an instance of more or less application independent manufacturing processes.

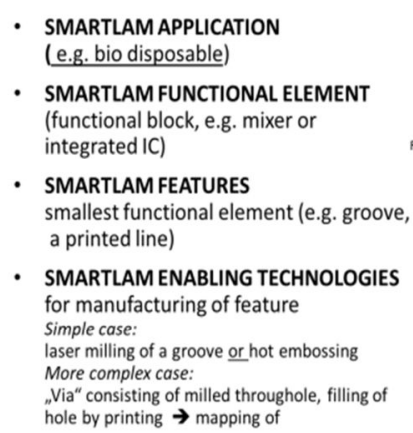

- Smartlam enabling teCHNologies for manufacturing of feature

Simple case:

laser milling of a groove or hot embossing

More complex case:

Via" consisting of milled throughole, filling of

hole by printing $\rightarrow$ mapping of

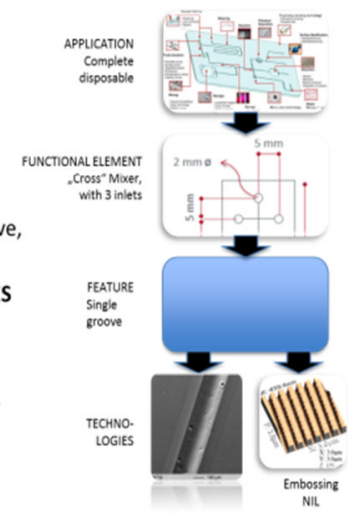

Fig. 3. SMARTLAM modeling hierarchies.

\subsection{Modelling of generic SMARTLAM functional elements}

This initial set of functional elements shall cover a broad variety of potential applications without being limited to the two main application fields, which are in the focus of SMARTLAM.

The requirements to the functional elements have to address the manufacturing methods inherent to SMARTLAM: They should either

- have the potential to become realised by a combination of the technologies available in one level (grooves, conductive tracks,...), or

- can be manufactured by a combination of layers with different properties, e.g. a valve, realised by an elastomer layer in between two rigid layers with circular cut outs.

Fig. 2 provides an example for a (hypothetic) disposable for bio analytics device, in which fluidic and electronic functional elements had been combined by an "intelligent" combination of layers of functionalised polymers with different material properties. Besides functionalised layers, e.g. for econtacting, layers with dedicated material properties or micro structured layers examples for integration of discrete parts are included, Positioning and contacting of a discrete chip or a battery widens the

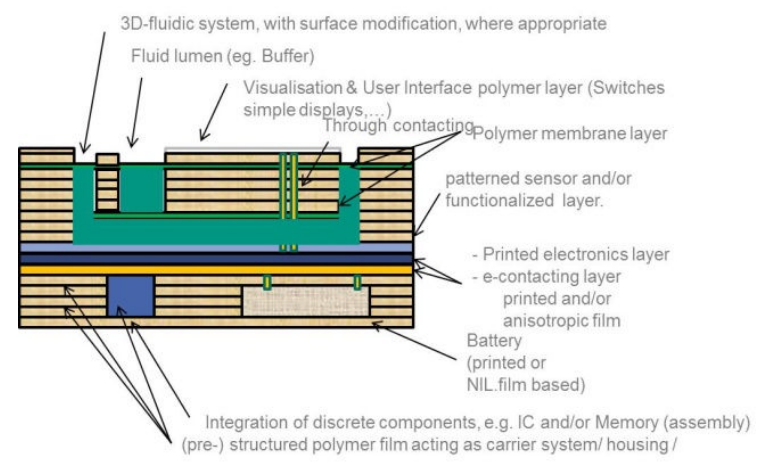

Fig. 2. SMARTLAM demonstrator for "intelligent combination of functionalized" polymer layers.

capabilities of the additive manufacturing capabilities.

In SMARTLAM the following types of functional elements for printed electronics had been identified over the first months and are currently subject to further investigations and discussions with respect to fulfill the application requirements as well as to meet the technological constraints:

\section{Printed electronics}

- $\quad$ electrode structures [line, interdigital]

- MR Sensors

- $\quad$ Capacitive switches

- Functional elements for the energy storage

- Printed batteries

- NIL-based Batteries

- $\quad$ Components for electronics

- Resistors

- Switches

\section{Functional elements for micro fluidics}

The concepts allows for a broad range of fluidic functional elements to be investigated in a more detail, especially in M7-12.

- Microfluidic channels

- Membranes

- Valves

- Mixers 


\section{- Storage}

- Actuators

Functional elements building on surface modification

SMARTLAM technologies basically allow for an active treatment of surface properties (chemical and physical properties). Surface activation (e.g. Ionisation) or an active control of wttability by laser processing are just two examples to mention in this context.

\section{Composite functional elements}

Some of the functional elements can be combined to composite functional elements of a "higher" integration level. The functional element "positioning and integration of a chip" may consist of two functional elements "milling of a pocket" and "econtacting"

\section{Implementation strategies}

Many of the functional elements mentioned above can be realized in a single manufacturing step, where the function can be realized by manufacturing of a single geometric element, which will be covered in more detail in the next paragraph. In most cases even more complex functional elements can be described as a combination of such features. From a process perspective however, there are typically multiple solution strategies for implementing functional elements. The "Via" functional element may serve as an example, as technological as well as chemical solutions are feasible and will have to be selected depending on the application boundary conditions. These solutions include but are not limited to:

- realisation of a "mechanical" contacting between film layers consisting of a through hole filled with electronic ink.

- Physical realisation of a contacting using the special material properties e.g. of "anisotropic conductive films"

\subsection{Features}

"Features" represent a kind of intermediate between the application-oriented function element and the "manufacturing output", mostly resulting in a set of geometric primitives that can be micro milled or cut, coated, printed, welded, etc.. The following examples may illustrate underlying concept:

- A single hole can be a vertical microfluidic channelas well as a pocket for integration of discrete parts.

- An array of such micro might represent a "micro sieve" for blood separation in the bio disposable application areas.

- A combination of the "hole"-feature with other features such as the filling of a hole with e-ink can result in a contacting for manufacturing of "vias", know from printed circuit board technology

Typical features:

- Channels (for fluidic or optical properties)

- Pockets (cavities, lumen or locating holes)

- Printed lines (conductive tracks, sensors, "via")
- Material layers with properties, different from polymers (realisation of batteries, realisation of membranes (elastomers)

Evaluation tests could demonstrate the validity of this approach and similar concepts on feature level had been successfully tested in other micro related contexts [3]. A thorough evaluation in order to validate the principles of this concept will be performed over the course of the project.

\section{Selection of manufacturing process chain and product design}

An integrated micro device often consists of a very large number of features which form the application oriented functional elements. For the fabrication of such devices a manufacturing process chain of high complexity is required. To address this problem, in SMARTLAM the product design and the process selection are incorporated into a hierarchical model where decisions are made at different abstraction layers. Similar hierarchical approaches have been elaborated and realised in CIM or STEP [4][5].

\subsection{Product design method}

In the SMARTLAM 3D-I concept the product designer uses a library of structural features which can be integrated to built functional elements. These features together with information about technologies that can be used for their fabrication are stored in a database. In the hierarchical model the first layer is the library and is the only layer open to the designer. The second layer is the pool of available technologies and forms the manufacturing process chain. The third layer is the process parameters and is used for the optimisation and selection of specific process chain (see Figure 4). If the simple example from the previous section is taken, the library will offer a variety of micro holes in a range of sizes and materials which can be arranged to form a micro sieve. Some of the technologies that can be used for the fabrication of wholes in a polymer material are micro milling, laser machining or micro- injection moulding. The process parameters for the machining of the specific holes selected by the designer are stored in the database. After the micro sieve the next functional element on the device might be a conductive line. Possible technologies for its execution would be any kind of lithography, aerosol or jet printing. The design can be continued with the next device feature or a lamination step. Thus is it is apparent that an effective method of selecting the best available chain has to be created. The advantage of using a library of preset geometries is that the designer is limited to structures which fabrication is already a well-established process. The number of alternative process depends on the completeness of the database which can be expanded when required. The addition of each new device feature multiplies the number of the possible manufacturing process chains by a factor equal to the number of available technologies for the feature. Therefore the development of fast and effective way of selecting the optimum chain is essential. 

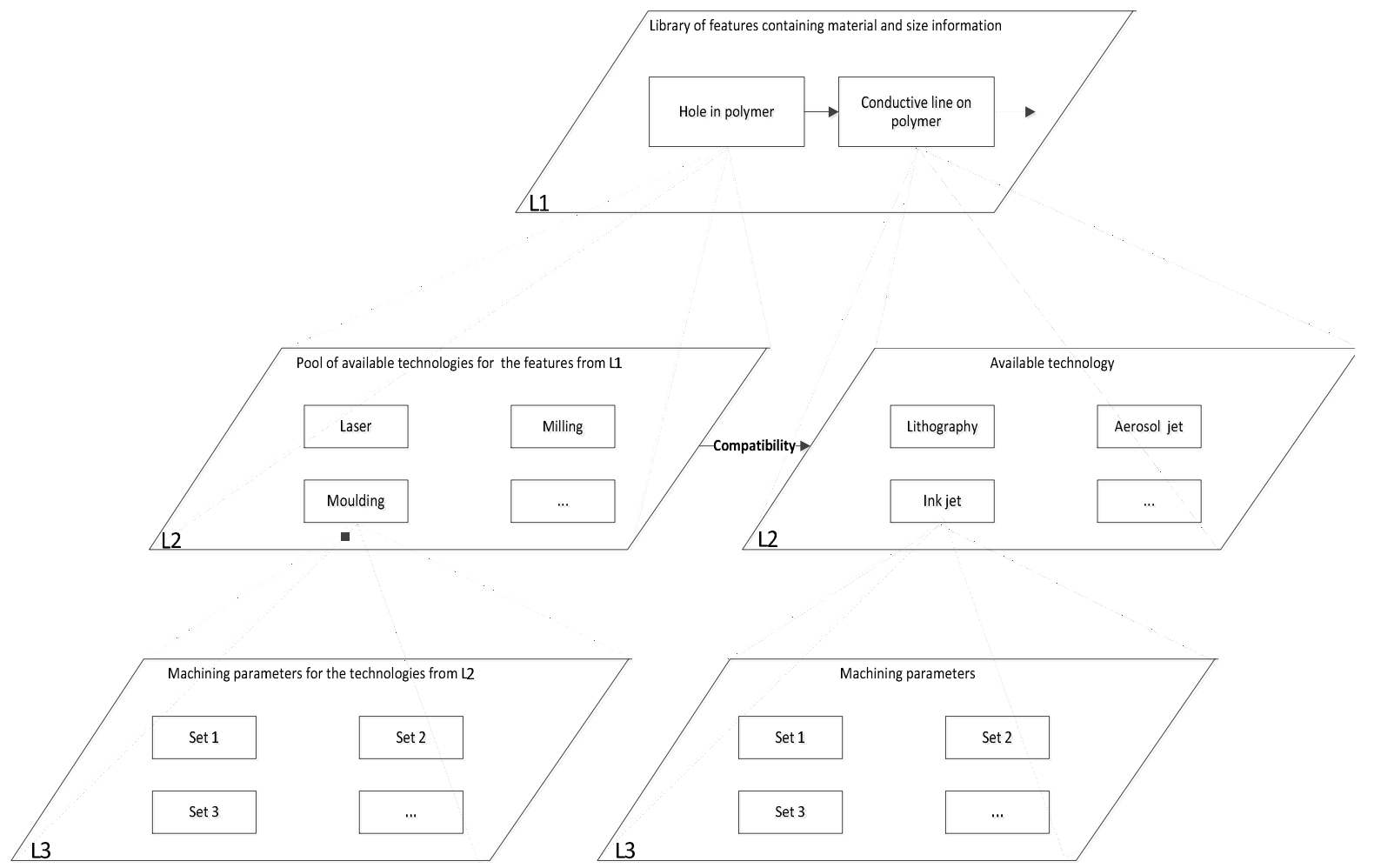

Fig. 4. SMARTLAM process sequence hierarchies.

\subsection{Process chain selection method}

Large number of the initially identified process chains are rejected by breaking some of the links on the second hierarchical level. This can be done either on material- technology (M-T) or technology- technology (T-T) compatibility criteria. (see Figure 4). The T-T compatibility criterion is relatively simple and normally there is a clear rejection or approval of a specific chain. In many cases this is the inability of combining vacuum with non- vacuum technologies. As an example, laser machining cannot be combined with electron lithography due to the extreme complications of the workpiece transfer. In cases when the technology compatibility assessment is not that straightforward SMARTLAM can exploit the Process Pair Interface Model developed under the EC FP7 funded EUMINAfab project which provides more detailed analysis of the pair maturity level [6]. The M$\mathrm{T}$ criterion is more complicated and requires some more detailed investigation. For many technologies there is a "favourite" material but this does not exclude the use of some other materials. For example PET is one of the most popular substrate materials for aerosol jet printing but shows poor UV laser machinability. This requires the development of a scoring system for different material- technology pairs and setting of threshold score below which the manufacturing chain is rejected.

After applying the compatibility criteria the remaining process chains are arranged in an array:

$$
\begin{aligned}
& \text { Array } \\
& =\left(\begin{array}{lllllll}
\operatorname{chain}_{1} & \cdots & \text { chain }_{i-1} & \text { chain }_{i} & \text { chain }_{i+1} & \cdots & \text { chain }_{n}
\end{array}\right)
\end{aligned}
$$

Technologies that can be associated with more than one set of process parameters are linked in a separate chain for each set. The minimisation of the array is executed in two phases. In the first one process parameters such as temperature or pressure are assessed and it is checked whether any of the technologies or materials in the chain imposes restrictions to these parameters. Chains which do not meet these criteria are rejected. The second minimisation phase is when the actual process optimisation is performed. This is done by comparing the process parameters against the user requirements. User requirements can be machining time, cost or accuracy. At the end only the best matching chain remains which is used for the fabrication of the designed device. An advantage of this approach is that the selection of the technologies also serves for identification of the process parameters. This allows for very fast and efficient reconfiguration of the SMARTLAM modules.

\section{Technologies and materials of relevance for the Smart manufacturing approach}

Representing the smallest building blocks in modelling of process chains three different types of technologies are currently under evaluation regarding their integration in the system setup:

- Technologies for additive manufacturing and eprinting aerosol jet printing. In SMARTLAM the eprinting functionality is realized by aerosol jet printing, offering good results for manufacturing of line-based geometries such as conductive paths [8]

- Handling assembly and bonding technologies [7]

- Technologies for direct and indirect milling and cutting of polymer films [laser milling, cutting, nano imprint lithography, hot embossing] 
Each of these technical sections will be briefly introduced with respect to its specific relevance for the Smartlam approach.

\subsection{Structuring Technologies}

For (pre-) structuring of films, technologies such as laser direct structuring but also indirect technologies such as NIL or hot embossing are under evaluation. Criteria to be mentioned in this context are scalability of processes, machine unit costs, required precision, small lot sizes vs. larger volumes.

First tests have been conducted at KIT-IAM to evaluate typical SMARTLAM features and functional elements. In SMARTLAM polymers such as polycarbonate (PC) or poly(methyl methacrylate) (PMMA) are used. Those polymers show a high laser beam absorption in the ultraviolet (UV) $(248 \mathrm{~nm}$, $193 \mathrm{~nm}$ ) as well as in the infrared (IR) $(10.6 \mu \mathrm{m})$. From previous studies it is known that a high optical absorption at UV wavelengths leads to low material removal rate, which in turn enables a precise control of the achieved ablation depth and a good surface quality [9]. With UV laser structuring lateral resolutions in the sub-micrometer range via direct writing or direct optical imaging of complex mask structures can be achieved. Furthermore below or in the range of the laser ablation threshold of the polymer a surface modification is possible. With optimized laser and process parameters surface modification can be applied in order to adjust the wettability of polymer surfaces from superhydrophilic to superhydrophobic properties.

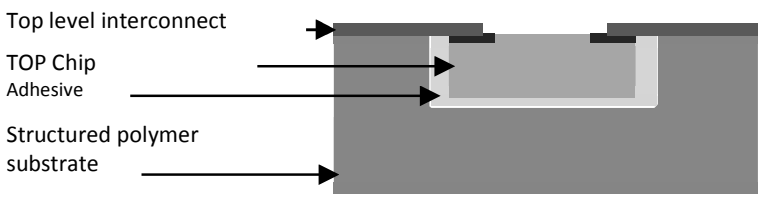

Fig. 5 Top Level interconnect design for Chip integration

IR laser structuring of polymers can provide a very flexible material processing with high processing speeds. High accuracy channel widths down to $50 \mu \mathrm{m}$ can be produced in PMMA. Nevertheless, due to the fact that IR laser-assisted ablation processing is mainly thermally driven, a main challenge is to achieve small structures $(<50 \mu \mathrm{m})$ with minimized heat affected zone.

An appropriate combination of UV and IR laser processing can be applied for the generation of functionalized polymeric micro devices [10]. Fig. 6 and Fig. 7 show laser structured thin polymer films using IR and UV laser systems.
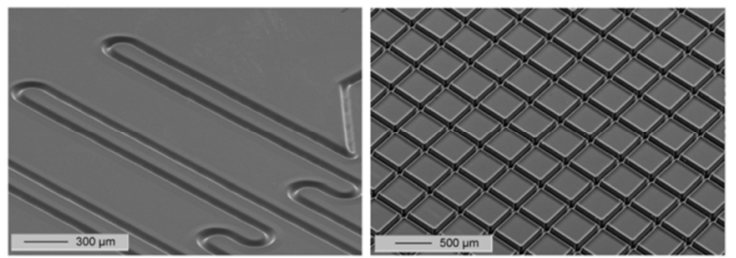

Fig. 6 Micro channel structures in PMMA achieved by IR laser ablation (wavelength: $\mathbf{1 0 . 6} \boldsymbol{\mu \mathrm { m } \text { ) }}$

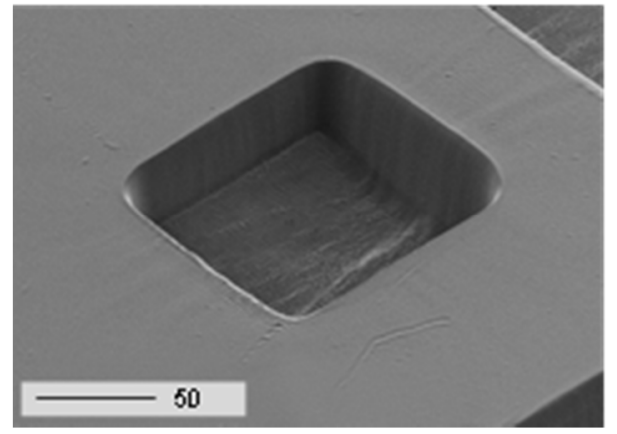

Fig. 7 Pocket structure in PET achieved by UV excimer laser ablation (wavelength $193 \mathrm{~nm}$ )

\subsection{Polymer film handling and assembly related technologies}

The polymer film handling addresses a limiting aspect with respect to the achievable precision: the handling and (fine) positioning of parts within the systems. Other technologies such as dispensing and bonding of polymer films are subject to a detailed analysis as well. While there exists a long experience in handling of polymer films the proper alignment of the sheet layers with respect to the required accuracy of a few $\mu \mathrm{m}$ is still challenging. Manufacturers have to deal with selective thermobonding to address the relaxation behaviour of the film material without loss in accuracy.

The polymer film handling process is shown in Fig. 8. The first step, feeding, supplies singular film sheets. This is challenging, as the films require to be provided as flat as possible for the precision pick and place process and have to be "loose" in order for the gripper to grab it. A magazine with an air suction for each sheet could be a possible approach.

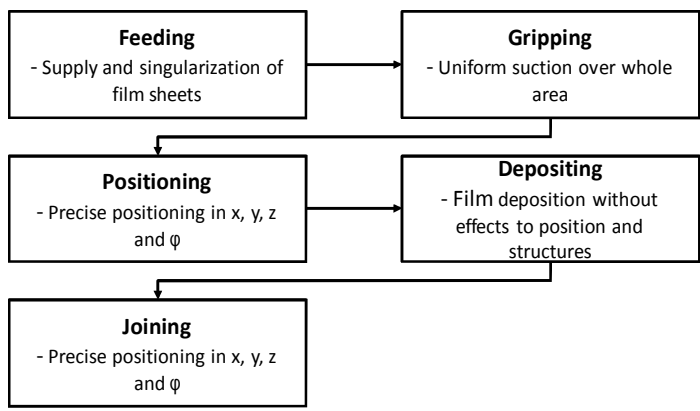

Fig. 8 Polymer film handling process

The second process step is gripping. As the film sheets are flexible, but still have to be precisely positioned, a uniform suction over the whole sheet area is pursued. Deformations during the gripping process have to be avoided at any cost, as these would lead to major deviations between the respective product layers. A flat vacuum gripper with an arrangement of holes $<<1 \mathrm{~mm}$ could be a possible approach.

The third step is the positioning of the sheet relative to the substrate. This requires positioning in position $(x, y, z)$ and orientation $(\varphi)$. The required precision depends on the type of layer. If the sheet features micro-structures, which require relative alignment to the substrate, the required positioning accuracy might be $<25 \mu \mathrm{m}$. If the sheet does not 
feature any micro-structures, the positioning accuracy is rather uncritical. A cartesian precision placement system with an additional rotational axis is proposed here.

Letting the sheet go is as critical and challenging as the gripping process. While it is very important to attach the sheet as uniformly as possible without any deformation, no deformation may happen during the disengagement. At the same time neither substrate nor sheet may be damaged during disengagement. A possible strategy could be a light blow-off of the sheet.

For the joining process several options exist, such as adhesive bonding, induction welding, laser welding, lamination or thermobonding [14]. The requirements to be addressed are joining of the sheet with the substrate without deformation, damage and voiding. Currently, a lamination process is investigated, as it seems to fit very well to the requirement profile.

For the integration of the functional elements "integration of discrete part" and the related technologies "milling of a pocket", "dispensing of glue" and "positioning of chip" first tests had been conducted to evaluate the feasibility.

The "milling of a pocket" is done by application of the above-mentioned structuring technologies. The further technologies are merged to a discrete part assembly process. For the functional element "integration of discrete part" first tests have been carried out with a top level interconnect design for chip integration (see Fig. 5.)

The chips have to be fixed in the cavities with adhesive bonding. In addition, the dispensed adhesive has to fulfill another important function: It works as a convex or concave bridge filling the gap between the LED and the substrate, on which electrical circuits can be printed. Furthermore the adhesive has to protect the chip to ensure reliability during operation. The length of the chips edge is smaller than $500 \mu \mathrm{m}$. The corresponding pockets are slightly larger. Therefore the amount of adhesive is in the low nanolitre range and has thus to be dispensed very precisely. Furthermore adhesive forces between the walls of the pocket and the adhesive disturb the accurate and repeatable positioning of the droplet. To still achieve a stable assembly process, high precision measuring equipment for the lateral and vertical dimensions of the pockets and the dispensed adhesive as well as a precision placement system with a positioning accuracy in the low micron range for the dispensing tool is required. The same high requirements have to be fulfilled for the chip placement. Fig. 9 shows optical measurements of three stages of the chip assembly process: at first the empty pocket is filled with adhesive and the assembled chips with the adhesive bridge.Afterwards the adhesive needs to be cured. The products assembled within SMARTLAM are based on polymer films. Currently a broad range of substrate materials is intended to be applied, e. g. polyethylene terephthalate (PET), polycarbonate (PC) or poly(methyl methacrylate) (PMMA). The glass transition temperatures (PET: $70^{\circ} \mathrm{C}$; PC: $145^{\circ} \mathrm{C}$; PMMA: $105^{\circ} \mathrm{C}$ ) should not be exceeded during the curing of the adhesive. For most common adhesives comparative high temperatures above $100{ }^{\circ} \mathrm{C}$ are needed for fast curing. To maintain low cycle times
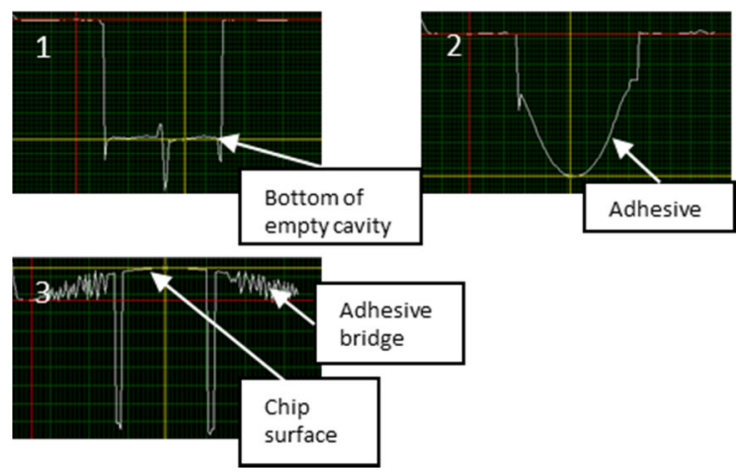

Fig. 9 Stages of chip assembly

with low temperatures UV curing adhesives are taken into account. Laser as part of the SMARTLAM system could potentially be used for precise and local curing to reduce the heat-affected zone compared to a convection oven.

The SMARTLAM e-printing functionality is used in a following step for the electrical connection of the chips.

4.3 Materials properties of specific relevance for SMARTLAM concept

Novel polymer film with advanced material properties are of a specific interest for the SMARTLAM approach. There exists a broad range of material features that will become of interest for SMARTLAM applications:

\section{"Standard materials"}

The use of polymer materials with dedicated optical and mechanical properties provides opportunities for a broad range of applications but does also cause problems while its application in the SMARTLAM context. While the activities in the startup phase are focussing on polymer films, the application of ceramic films, similar to LTCC applications [15] as well as the application of flexible glass [16] but also combinations of different substrate layers with surface properties will be subject to the future research.

In addition materials with advanced chemical and physical properties will be evaluated in later stage of the project. As an outlook may serve the following materials:

- Anisotropic conductive films allowing for the an electrical conductivity vertical to the sheet plane, While the application in LCD panel production and chip industry is well established the application of the ACF in flexible environment is still subject to research activities, e.g. in the field of flip-chip on flex packages assembly [11]

- $\quad$ high optical transparency, robust flexibility, and excellent conductivity caused by general synthesis of aligned carbon nanotube/polymer composite films with. (potential applications such as flexible conductors for optoelectronic devices) [12]

- $\quad$ Actuation of the microsystem, caused by electro active properties of the polymer [13] 


\subsection{System integration on process chain level}

According to the 3D-integration paradigm different combinations of process sequences -each representing the respective process sequences for manufacturing of the respective design building blocks. Figure 10 provides an example how such process sequences could look like

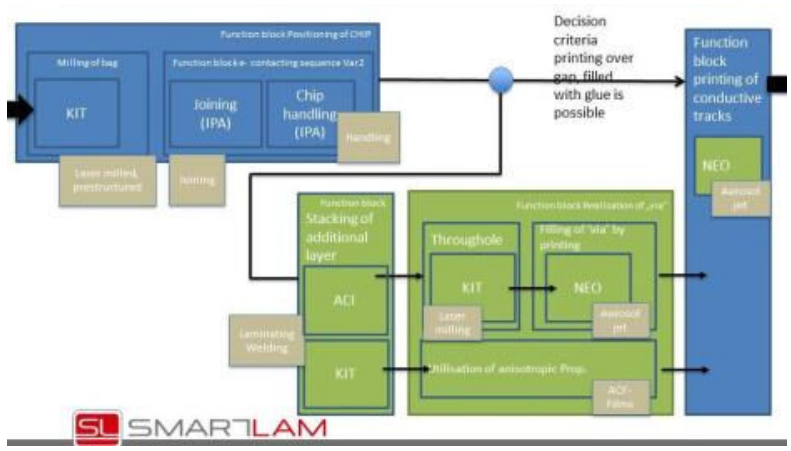

Fig. 10 Example of a process sequence for connecting a discrete part

Different setups are currently under evaluation and testing, contributing to the decision support tools as well as the database.

\section{Conclusions and Outlook}

In the paper a novel approach for flexible scalable manufacturing of micro components building on a combination of laminated objects modelling, integration of laser technologies for structuring of films and printed electronics for e-contacting and printing of electronic components was presented.

Specific attention was paid to the design of applications, building on a new approach for 3Dintegration, (3D-I). Design elements on different aggregation levels and their implementation have been introduced.

Over the next month these concepts will be developed further and adapted to the needs of the two project demonstrators in the field of lighting and microfluidics

\section{Acknowledgements}

The research related to the SMARTLAM flexible scalable manufacturing approach receives funding from the European Community's Seventh Framework Programme (FP7/2007-2013) Grant agreement No. 314580.

Finally, the support by the Karlsruhe Nano Micro Facility (KNMF, www.kit.edu/knmf) for laser processing is gratefully acknowledged.

\section{References}

[1] Muellera,B., Kochan, D.; Laminated object manufacturing for rapid tooling and patternmaking in foundry industry, Computers in Industry, Volume 39, Issue 1, June 1999, Pages 47-53
[2] Schwartz, E. Cornell University MSE, 2006; http://people.ccmr.cornell.edu/ cober/mse542/page2/fil es/Schwartz\%20R2R\%20Processing.pdf

[3] Albers, A., Börsting, P., Wildermuth, P; Haußelt, J, Kraft, O. Kolloquium Mikroproduktion und Abschlusskolloquium SFB 499 ; 11. - 12. Oktober 2011, Karlsruhe Kraft, O., Haug A., Vollertsen, F., Büttgenbach S [Hrsg.]; (KIT Scientific Reports ; 7591), KIT scientific Publishing

[4] Xun Xu,Andrew Yeh Chris Nee, Advanced Design and Manufacturing Based on Step, Springer, 2009

[5] Dickerhof, M., Mampel, U. DIdic, M; Workflow and CIMOSA, a background case study; Computers in Industry 40 _1999. 197-205, Elesevier science, 1999

[6] He, X.; Gao, F.; Tu, G.; Hasko, D.; Huettner, S.; Steiner, U.;Greenham, N. C.; Friend, R. H.; Huck, W. T. S. Formation of Nanopatterned Polymer Blends in Photovoltaic Devices. Nano Lett. 2010, 10, 1302-1307.

[7] He, X: Gao, F.; Tu, G.; Hasko, D.; Huettner, S. Steiner, U.;Greenham, N. C.; Friend, R. H.; Huck, W. T. S. Formation of Nanopatterned Polymer Blends in Photovoltaic Devices. Nano Lett. 2010, 10, 1302-1307.

[8] King, B. and Renn, M.; Aerosol jet direct write printing for mil aero electronic applications: Source: Optomec, Inc., 3911 Singer, NE, Albuquerque, NM 87109, USA.

[9] Pfleging, W., Kohler, R., Südmeyer, I., Rohde, M.; Laser Micro and Nano Processing of Metals, Ceramics , and Polymers; in: "Laser-Assisted Fabrication of Materials", Springer Series in Materials Science Volume 161, 2013, pp 319-374

[10] Pfleging, W., Kohler, R., Schierjott, P., Hoffmann, W.; Laser patterning and packaging of CCD-CE-Chips made of PMMA, Sensors and Actuators B: Chemical, Volume 138, Issue 1, 24 April 2009, Pages 336-343).

[11] Chan, Y.C., Luk, D.Y.,; Effects of bonding parameters on the reliability performance of anisotropic conductive adhesive interconnects for flip-chip-on-flex packages assembly II. Different bonding pressure,in Microelectronics Reliability, Volume 42, Issue 8, August 2002, Pages 1195-1204, Elsevier, 2002

[12] Peng, Huisheng; Aligned Carbon Nanotube/Polymer Composite Films with Robust Flexibility, High Transparency, and Excellent Conductivity Division of Materials Physics and Applications, Los Alamos National Laboratory, Los Alamos, New Mexico 87545 J. Am. Chem. Soc., 2008, 130 (1), pp 42-43 DOI: 10.1021/ja078267m

[13] Bar-Cohen, Y.; Electroactive Polymer (EAP) Actuators as Artificial Muscles: Reality, Potential, and Challenges, SPIE ebooks, Second Edition, 2004 http://ebooks.spiedigitallibrary.org/book.aspx?bookid=1 46 [05.04.2013]

[14] Tahhan, I; "Ein Beitrag zum wirtschaftlichen Fügen von mikrofluidischen Baugruppen," Universitätsbibliothek Freiburg, Dissertation, 2009

[15] http://www.schott.com/epackaging/german/ download/schott_db_Itcc_via_rz_d_2012_03_29indd.p df, as of April, 6th 2013

[16] http://www.convertingquarterly.com/Portals/ 1/files/matteucci-awards/2011-Flexible-GlassSubstrates-for-R2R-Manufacturing.pdf as of April, 6th 2013 